Louisiana State University

LSU Digital Commons

Faculty Publications

Department of Physics \& Astronomy

8-1-2011

\title{
Evidence for pre-existing dust in the bright type IIn SN2010jI
}

\author{
J. E. Andrews \\ Louisiana State University \\ Geoffrey C. Clayton \\ Louisiana State University \\ R. Wesson \\ University College London \\ B. E.K. Sugerman \\ Goucher College \\ M. J. Barlow \\ University College London
}

See next page for additional authors

Follow this and additional works at: https://digitalcommons.Isu.edu/physics_astronomy_pubs

\section{Recommended Citation}

Andrews, J., Clayton, G., Wesson, R., Sugerman, B., Barlow, M., Clem, J., Ercolano, B., Fabbri, J., Gallagher, J., Landolt, A., Meixner, M., Otsuka, M., Riebel, D., \& Welch, D. (2011). Evidence for pre-existing dust in the bright type IIn SN2010jl. Astronomical Journal, 142 (2) https://doi.org/10.1088/0004-6256/142/2/45

This Article is brought to you for free and open access by the Department of Physics \& Astronomy at LSU Digital Commons. It has been accepted for inclusion in Faculty Publications by an authorized administrator of LSU Digital Commons. For more information, please contact ir@lsu.edu. 


\section{Authors}

J. E. Andrews, Geoffrey C. Clayton, R. Wesson, B. E.K. Sugerman, M. J. Barlow, J. Clem, B. Ercolano, J. Fabbri, J. S. Gallagher, A. Landolt, M. Meixner, M. Otsuka, D. Riebel, and D. L. Welch 


\title{
EVIDENCE FOR PRE-EXISTING DUST IN THE BRIGHT TYPE IIn SN 2010j1
}

\author{
J. E. Andrews ${ }^{1}$, Geoffrey C. Clayton ${ }^{1}$, R. Wesson ${ }^{2}$, B. E. K. Sugerman ${ }^{3}$, M. J. Barlow ${ }^{2}$, J. Clem ${ }^{1}$, B. Ercolano ${ }^{4}$,

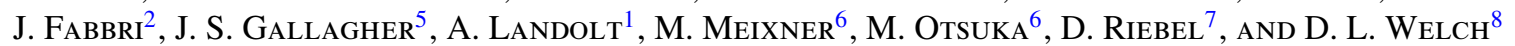 \\ ${ }^{1}$ Department of Physics and Astronomy, Louisiana State University, 202 Nicholson Hall, Baton Rouge, LA 70803, USA; \\ jandrews@phys.1su.edu, landolt@phys.1su.edu,gclayton@fenway.phys.1su.edu, jclem@phys.lsu.edu \\ ${ }^{2}$ Department of Physics and Astronomy, University College London, Gower Street, London WC1E 6BT, UK; \\ mjb@star.ucl.ac.uk, jfabbri@star.ucl.ac.uk, rwesson@star.ucl.ac.uk \\ ${ }^{3}$ Department of Physics and Astronomy, Goucher College, 1021 Dulaney Valley Road, Baltimore, MD 21204, USA; ben.sugerman@goucher.edu \\ ${ }^{4}$ Ludwig-Maximilians-Universitaet, University Observatory Munich (USM), \\ Scheinerstr 1, D-81679 Muenchen, Germany; ercolano@usm.uni-muenchen.de \\ ${ }^{5}$ Department of Mathematics, Physics, and Computer Science, Raymond Walters College, 9555 Plainfield Road, \\ Blue Ash, OH 45236, USA; gallagil@ucmail.uc.edu \\ ${ }^{6}$ Space Telescope Science Institute, 3700 San Martin Drive, Baltimore, MD 21218, USA; meixner@stsci.edu, otsuka@stsci.edu \\ ${ }^{7}$ Department of Physics and Astronomy, Johns Hopkins University, 3400 North Charles Street, Baltimore, MD 21218, USA; driebel@ pha.jhu.edu \\ ${ }^{8}$ Department of Physics and Astronomy, McMaster University, 1280 Main Street West, Hamilton, ON L8S 4M1, Canada; welch@ physics.mcmaster.ca \\ Received 2011 April 13; accepted 2011 June 2; published 2011 June 30
}

\begin{abstract}
SN 2010jl was an extremely bright, Type IIn supernova (SN) which showed a significant infrared (IR) excess no later than 90 days after explosion. We have obtained Spitzer 3.6 and $4.5 \mu \mathrm{m}$ and JHK observations of SN 2010j1 $\sim 90$ days post-explosion. Little to no reddening in the host galaxy indicated that the circumstellar material lost from the progenitor must lie in a torus inclined out of the plane of the sky. The likely cause of the high mid-IR flux is the reprocessing of the initial flash of the $\mathrm{SN}$ by pre-existing circumstellar dust. Using a three-dimensional Monte Carlo radiative-transfer code, we have estimated that between 0.03 and $0.35 M_{\odot}$ of dust exists in a circumstellar torus around the SN located $6 \times 10^{17} \mathrm{~cm}$ away from the SN and inclined between $60^{\circ}$ and $80^{\circ}$ to the plane of the sky. On day 90 , we are only seeing the illumination of approximately $5 \%$ of this torus, and expect to see an elevated IR flux from this material up until day $\sim 450$. It is likely this dust was created in a luminous blue variable (LBV) like mass-loss event of more than $3 M_{\odot}$, which is large but consistent with other LBV progenitors such as $\eta$ Carinae.
\end{abstract}

Key words: circumstellar matter - dust, extinction - supernovae: general - supernovae: individual (SN 2010jl)

Online-only material: color figures

\section{INTRODUCTION}

SN 2010jl was the brightest core-collapse supernova (CCSN) of 2010 , with a peak unfiltered brightness of 12.9 mag, and a corresponding peak absolute magnitude of roughly -20 (Newton $\&$ Puckett 2010). A spectrum taken on 2010 November 5 was classified as a Type IIn (Benetti et al. 2010), a particular class of Type II supernovae $(\mathrm{SNe})$ which show narrow $\left(\sim 100 \mathrm{~km} \mathrm{~s}^{-1}\right)$ and often intermediate $\left(\sim 1000 \mathrm{~km} \mathrm{~s}^{-1}\right)$ lines in $\mathrm{H}$ and $\mathrm{He}$ along with normal broad $\left(10,000 \mathrm{~km} \mathrm{~s}^{-1}\right)$ lines due to the expansion of the ejecta (Schlegel 1990). The narrow emission lines in their spectra are attributed to the ionization of the pre-existing circumstellar material (CSM) which has been excited by the initial flash of the SN. Intermediate lines can arise at early times due to the high optical depth in the CSM, which can cause multiple scatterings from thermal electrons that result in the broadening of the narrow lines (Smith et al. 2010). It is believed the progenitors of Type IIn SNe, which constitute 9\%-10\% of the total population of CCSNe (Smith et al. 2011a; Li et al. 2011; Smartt et al. 2009), lose $10^{-4}$ to $10^{-2} M_{\odot} \mathrm{yr}^{-1}$ (Fox et al. 2011; Kiewe et al. 2010) orders of magnitude more material than normal Type II progenitors. Pre-discovery Hubble Space Telescope images indicate that the progenitor of SN 2010jl was likely a massive star, $>30 M_{\odot}$, which could have been a luminous blue variable (LBV) in outburst phase (Smith et al. 2011b).

SN 2010jl is only the second CCSN progenitor with a mass greater than $17 M_{\odot}$ detected, the other being the LBV progenitor of SN 2005gl (Gal-Yam \& Leonard 2009; Gal-Yam et al. 2007). This is important for the study of dust production around CCSNe since the $\mathrm{SNe}$ from less massive progenitors $\left(8-17 M_{\odot}\right)$ only seem to be producing $10^{-2}-10^{-4} M_{\odot}$ of dust (Elmhamdi et al. 2003; Sugerman et al. 2006; Meikle et al. 2007; Kotak et al. 2009; Andrews et al. 2010, for example). This is far less than the $\sim 0.1-1 M_{\odot}$ of dust estimated per CCSNe needed to explain the large amounts of dust seen in galaxies of $z>6$. This has led to speculation that more massive progenitors existed among the Population III stars of high- $z$ galaxies, which may produce more dust (Cherchneff \& Dwek 2010, for example). To date though, there is indirect evidence that some nearby dustproducing CCSNe have progenitors more massive than $17 M_{\odot}$, for example, SN 2007it (Andrews et al. 2011), and are also only producing dust masses of $\sim 10^{-3} M_{\odot}$. This may indicate that the amount of dust produced is independent of progenitor mass. The study of dust formation in SN 2010jl, which likely has a massive progenitor, will provide an opportunity to explore this relationship more fully.

Due to the nature of Type IIn SNe, we expect pre-existing CSM gas and dust to exist around the SN. Therefore, to distinguish newly formed dust from dust that was already present, it is imperative to take early-time observations to measure the contribution from pre-existing dust to serve as a baseline for later times when conditions may be favorable for new dust condensation. Studying the pre-existing dust around CCSNe can also be essential to understanding the progenitor properties. Continued observations of the interaction between 


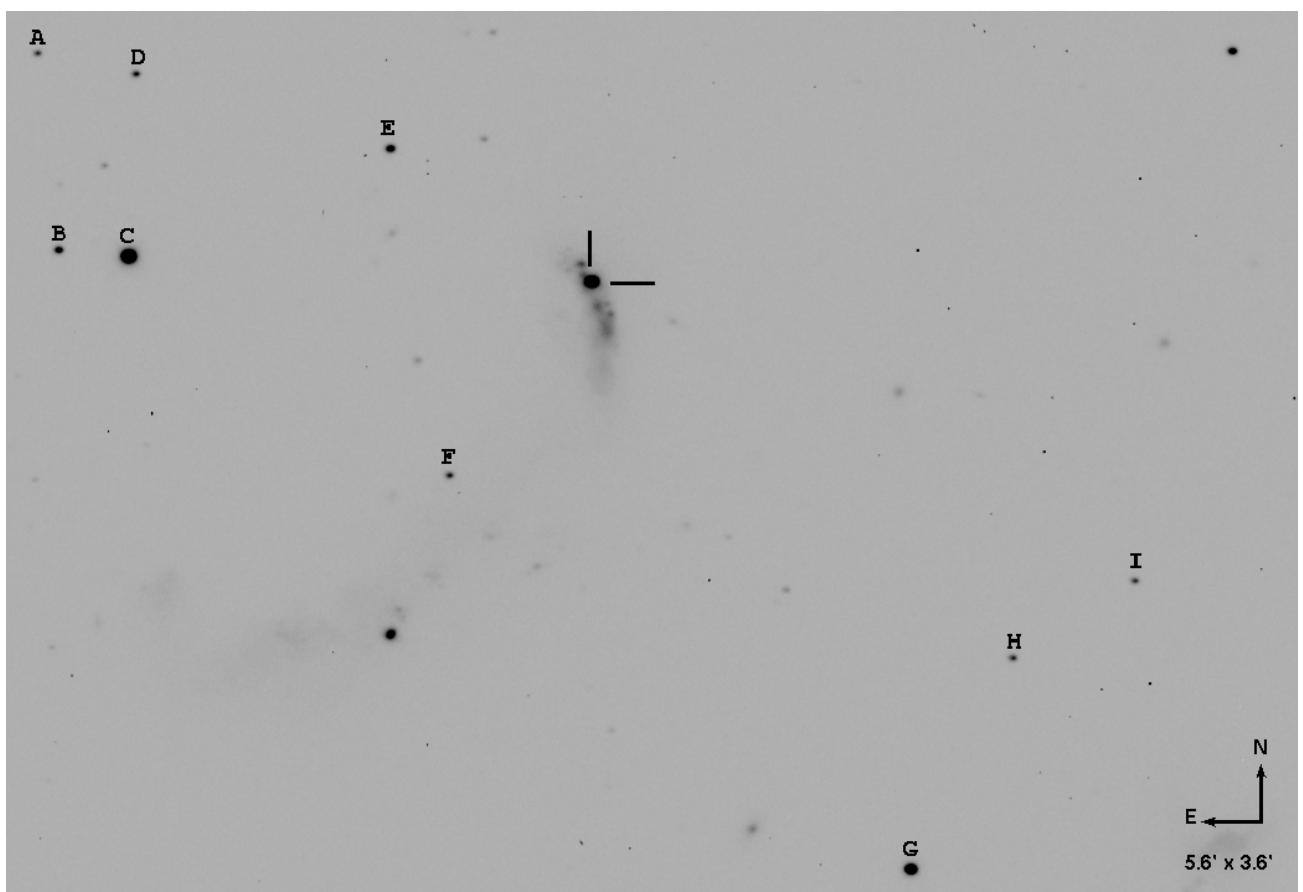

Figure 1. $V$-band finder chart for tertiary standards for the field around UGC 5189 on 2011 April 5. Magnitudes are listed in Table 1.

Table 1

Tertiary BVRI Standards for UGC 5189

\begin{tabular}{lccccc}
\hline \hline Star & $U$ & $B$ & $V$ & $R$ & $I$ \\
\hline A & $20.553 \pm 0.1351$ & $19.611 \pm 0.0453$ & $18.486 \pm 0.0180$ & $17.765 \pm 0.0148$ & $17.152 \pm 0.0366$ \\
B & $19.087 \pm 0.0429$ & $18.468 \pm 0.0176$ & $17.513 \pm 0.0123$ & $16.963 \pm 0.0084$ & $16.488 \pm 0.0200$ \\
C & $14.929 \pm 0.0138$ & $14.677 \pm 0.0036$ & $13.944 \pm 0.0049$ & $13.521 \pm 0.0048$ & $13.131 \pm 0.0038$ \\
D & $19.807 \pm 0.3422$ & $19.470 \pm 0.0508$ & $18.164 \pm 0.0138$ & $17.313 \pm 0.0167$ & $16.606 \pm 0.0153$ \\
E & $19.967 \pm 0.0636$ & $18.751 \pm 0.0537$ & $17.189 \pm 0.0065$ & $16.062 \pm 0.0111$ & $14.685 \pm 0.0052$ \\
F & $19.888 \pm 0.0995$ & $19.456 \pm 0.0448$ & $18.212 \pm 0.0201$ & $17.511 \pm 0.0154$ & $16.823 \pm 0.0450$ \\
G & $16.986 \pm 0.0157$ & $16.323 \pm 0.0060$ & $15.405 \pm 0.0027$ & $14.877 \pm 0.0059$ & $14.427 \pm 0.0055$ \\
H & $18.965 \pm 0.0834$ & $19.022 \pm 0.0597$ & $18.310 \pm 0.0162$ & $17.848 \pm 0.0220$ & $17.504 \pm 0.0274$ \\
I & $20.439 \pm 0.3093$ & $19.566 \pm 0.1121$ & $18.433 \pm 0.0174$ & $17.685 \pm 0.0211$ & $17.053 \pm 0.0334$ \\
\hline
\end{tabular}

the SN and the surrounding CSM material over the first few years can reveal valuable information about densities, velocities, and overall mass-loss history of the progenitor as well as progenitor mass itself (Fox et al. 2011; Smith et al. 2009, and references therein). This paper on SN 2010jl presents a spectral energy distribution (SED) using optical, near-, and mid-infrared photometry of SN 2010jl obtained $\sim 90$ days after explosion and estimates of the mass, size, and age of the circumstellar dust.

\section{OBSERVATIONS}

Although the discovery date of SN 2010jl was 2010 November 3.5 (Newton \& Puckett 2010), comparison of the optical spectra with other similar SNe (Yamanaka et al. 2010) as well as pre-discovery images (Stoll et al. 2010) indicate that the explosion date was likely early October. An ASAS image taken on 2010 October 9.6 shows the $\mathrm{SN}$ with a $V$ magnitude of 13.79 (Stoll et al. 2010). This is consistent with the GELATO modeling of SN 2010jl to SN 2006tf, which indicated that it was about a month past explosion (Yamanaka et al. 2010), and has led us to adopt 2010 October 10 (JD 2455480) as the date of explosion throughout this paper. It is located in the interacting galaxy UGC 5189A, for which throughout this paper we adopt the distance of $48.9 \mathrm{Mpc}$ (Smith et al. 2011b). We have also assumed a Galactic foreground reddening of $E(B-V)=0.027$ from Schlegel et al. (1998), and are assuming zero reddening in the host galaxy based on the previous estimates for SN 2010j1 by Patat et al. (2011) and Smith et al. (2011b).

A UVBRI photometric sequence of the UGC 5189A field (listed in Table 1 and shown in Figure 1) was taken on the night of 2010 December 5 (JD 2455504) at the KPNO $2.1 \mathrm{~m}$ telescope. These tertiary standards were calculated using the same methods as Andrews et al. (2010, 2011). The processing of all images to remove instrumental signatures was accomplished using the standard techniques of subtracting a median-filtered bias frame and dividing by master twilight flat-field images for each filter. Also, the fringing effects in the $I$-band exposures were removed by scaling and subtracting a master fringe frame from the program images. In order to transform the instrumental magnitudes to the standard system, between 30 and 35 different stars (depending on filter) were observed from the lists of Landolt (2009) in addition to the SN 2010jl field. These stars were observed both near zenith and at a high airmass $(\sim 1.75)$ and were selected due to their color ranges. Thus, they provided a viable sample of stars from which to derive accurate color and extinction coefficients as well as magnitude zero-points for each filter. The resulting rms scatter of the residuals from the 


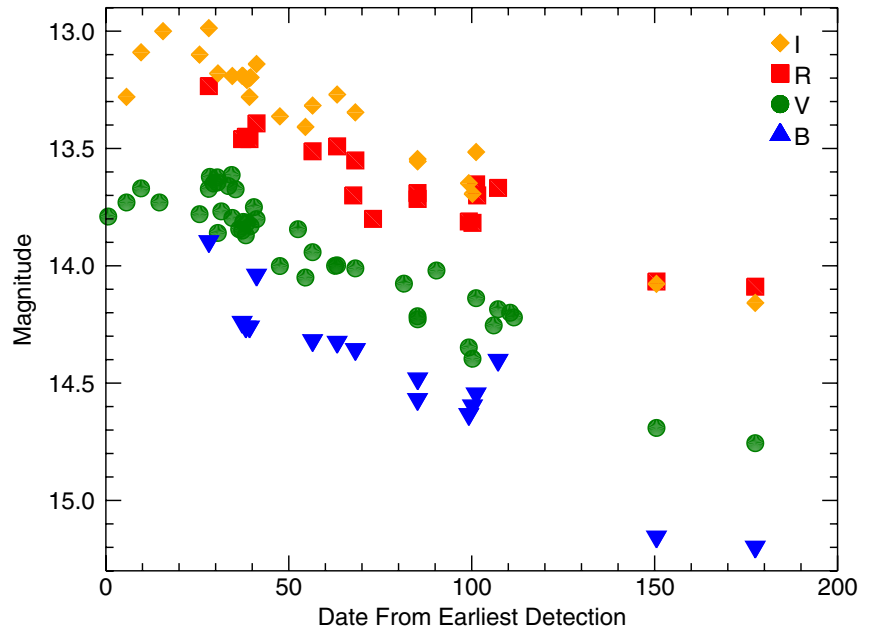

Figure 2. Optical light curve of SN 2010jl. Data are from AAVSO, Stoll et al. (2010), and new data presented in Table 2 in this paper.

(A color version of this figure is available in the online journal.)

Table 2

BVRI Observations of SN 2010j1

\begin{tabular}{lcccccc}
\hline \hline JD & Age & $B$ & $V$ & $R$ & $I$ & Telescope/Instrument \\
\hline 2455535 & 55 & 14.32 & 13.94 & 13.51 & 13.32 & KPNO/1.2 m \\
& & \pm 0.01 & \pm 0.01 & \pm 0.01 & \pm 0.01 & \\
2455629 & 149 & 15.16 & 14.69 & 14.07 & 14.08 & KPNO $/ 1.2 \mathrm{~m}$ \\
& & \pm 0.01 & \pm 0.02 & \pm 0.01 & \pm 0.01 & \\
2455656 & \multirow{2}{*}{176} & 15.20 & 14.76 & 14.09 & 14.16 & KPNO $/ 1.2 \mathrm{~m}$ \\
& & \pm 0.03 & \pm 0.01 & \pm 0.01 & \pm 0.01 & \\
\hline
\end{tabular}

transformations revealed that the photometry for selected stars in the SN 20010jl field derived from this night's observations is accurate at the $1 \%-2 \%$ level depending on filter. BVRI photometry was obtained either from observations from the KPNO $1.2 \mathrm{~m}$ (Table 2), the literature (Stoll et al. 2010) or via the AAVSO data server and is plotted in Figure 2. The data shown in the plot have not been corrected for foreground extinction. For the SED we used an observation from 2011 January 3 (JD 2455563), day 88, submitted to the AAVSO by contributor Etienne Morelle (observations from the AAVSO International Database 2010, private communication).

$J H K_{s}$ observations of SN 2010jl were taken on 2011 January 21 (JD 245583), or day 108, with the WHIRC camera on the WIYN telescope (Meixner et al. 2008). The detector offers a pixel scale of $0^{\prime \prime} .098$ pixel $^{-1}$, and a field of view of $3^{\prime} \times 3^{\prime}$. All images were taken in dither mode and aligned and stacked prior to flat fielding and bias correction. Eleven frames of $120 \mathrm{~s}$ were taken in the $J$ band, 12 frames of $120 \mathrm{~s}$ in the $H$ band, and 13 frames of $90 \mathrm{~s}$ in the $K$ band were taken of the SN 2010jl field. The transformation from instrumental to $J H K_{s}$ magnitudes was accomplished using Two Micron All Sky Survey (2MASS) stars located in the frame for comparison photometry. Uncertainties were calculated by adding in quadrature the uncertainties associated with the 2MASS comparison stars and the uncertainties in the point-spread function (PSF) photometry. The photometry is listed in Table 3 and shown in Figure 3.

The Spitzer IRAC (3.6 and $4.5 \mu \mathrm{m})$ images taken on 2011 January 5 (JD 2455565), corresponding to day 90, were mosaicked and resampled using standard MOPEX procedures to improve photometric quality. Pre-explosion IRAC images of UGC 5189A were also available in the Spitzer archive from 2007 December 27 (Program 40301; PI: Fazio) which were used to subtract from the SN 2010jl images to get accurate photometry. PSF photometry was performed using the position specific point-response function images. Table 3 contains the measured mid-infrared (mid-IR) fluxes obtained from Spitzer. Figure 3 presents the SED of SN 2010jl on $\sim$ day 90 (88 for $B V R I$ and 108 for $J H K)$. Statistical uncertainties presented in the plot represent $1 \sigma$ errors. The data shown in Figure 3 have been corrected for foreground extinction of $E(B-V)=0.027$ (Schlegel et al. 1998).

\section{DISCUSSION}

The SED of SN 2010j1, shown in Figure 3, shows an IR excess on day 90 which indicates the presence of warm dust around the SN. The IR excess could be explained by two possible scenarios, one being that pre-existing CSM dust existing beyond the evaporation radius is reprocessing the initial flash of the $\mathrm{SN}$ into the infrared. Another possibility, although less likely, is that there is already dust forming in a cool dense shell (CDS) that has formed between the forward and reverse shocks created between the ejecta and the pre-existing CSM gas interaction (Chevalier \& Fransson 1994; Pozzo et al. 2004). SN 2006jc (Smith et al. 2008) and SN 2005ip (Smith et al. 2009; Fox et al. 2009) formed dust at day 50 and 75, respectively, due to interaction with the CSM. This newly formed dust would also create an IR excess. Without optical spectra taken at the same epoch to look for the emergence of asymmetries, we cannot clearly distinguish between pre-existing and newly formed dust.

The progenitor star of a CCSN is expected to have lost massive amounts of gas and dust prior to explosion, which will exist in the CSM. When the star becomes an SN, the initial UV flash from the explosion will evaporate the dust grains a certain distance from the SN, dependent on the initial luminosity. According to Dwek (1983, 1985) and Fox et al. (2009) an SN with an initial luminosity of $1 \times 10^{10} L_{\odot}$ will clear a cavity with a radius $r_{\text {evap }}=6 \times 10^{16} \mathrm{~cm}$ if the CSM is carbon rich or $r_{\text {evap }}=3 \times 10^{17} \mathrm{~cm}$ if the CSM is oxygen rich. Assuming the maximum luminosity of SN 2010jl was $3 \times 10^{43} \mathrm{erg} \mathrm{s}^{-1}$ $\left(M_{v}=-20\right)$, this is roughly $10^{10} L_{\odot}$, we can expect a cavity of cleared material around the $\mathrm{SN}$ to have a radius of approximately the same size.

Using blackbody fits (shown in Figure 3), we made initial estimates of $R_{\text {in }}$ (the inner radius of the shell), luminosity, and temperature to constrain the model. These yielded a dust

Table 3

IR Observations of SN 2010j1

\begin{tabular}{lccccccc}
\hline \hline JD & Age & $J$ & $H$ & $K_{s}$ & $3.6 \mu \mathrm{m}$ & $4.5 \mu \mathrm{m}$ & Telescope/Instrument \\
\hline 2455565 & 90 & & & & $4.04 \mathrm{mJy}$ & $4.52 \mathrm{mJy}$ & Spitzer $/$ IRAC \\
& & & & & \pm 0.14 & \pm 0.18 & \\
2455583 & \multirow{2}{*}{108} & 13.41 & 13.09 & 13.75 & & & WIYN/WHIRC \\
& & \pm 0.2 & \pm 0.2 & \pm 0.2 & & & \\
\hline
\end{tabular}




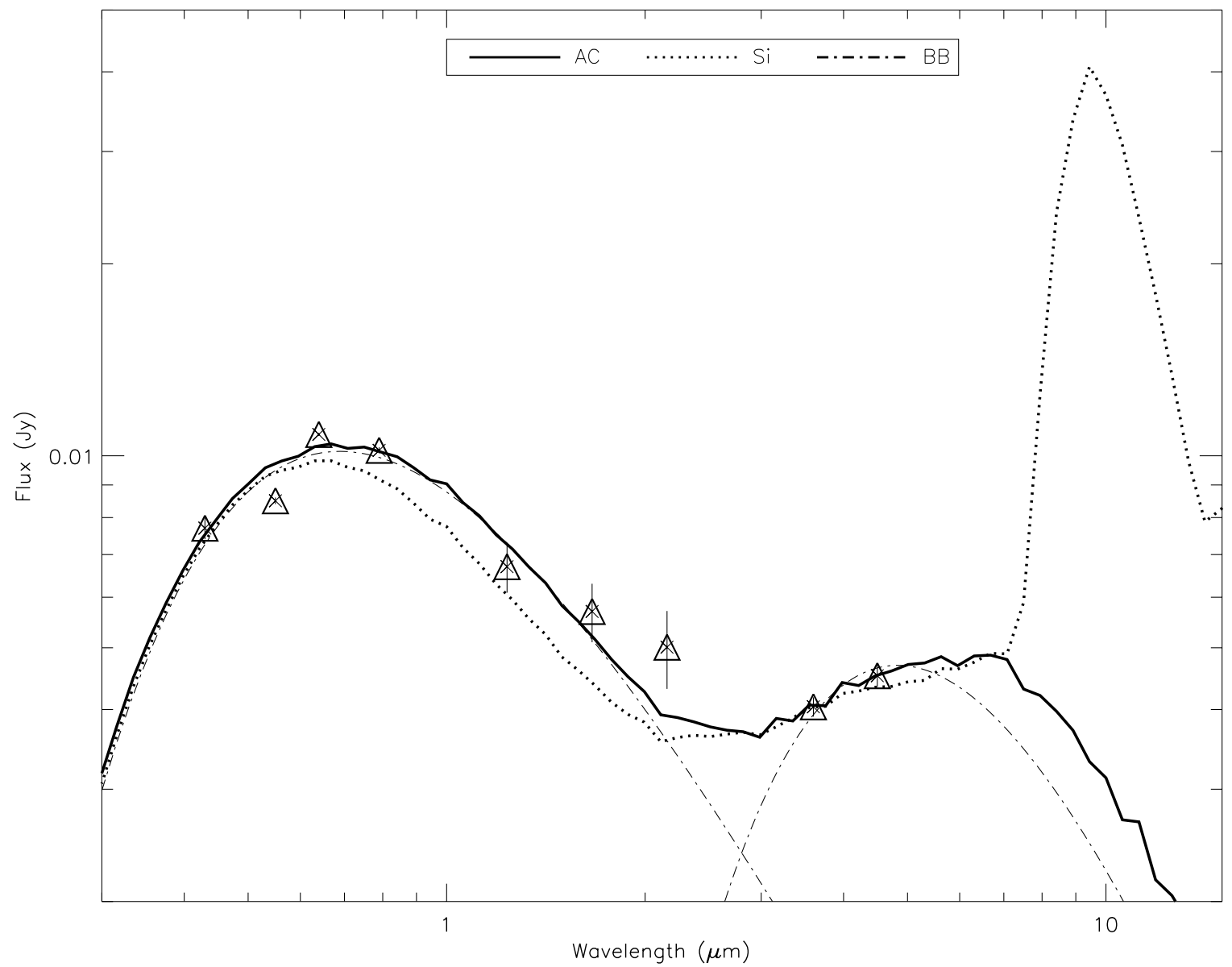

Figure 3. SED of SN 2010j1 on day 90. IR photometry is listed in Table 3, and optical photometry was obtained from the AAVSO, specifically contributor Etienne Morelle. Dash-dotted lines are the blackbody fits to the data. The solid line indicates the $100 \%$ amorphous carbon inclined at $60^{\circ}$ MOCASSIN torus model fit, the dotted line is the same except for the composition being $100 \%$ silicates. As the figure shows, without observations longward of $4.6 \mu \mathrm{m}$, we cannot accurately constrain the dust composition.

temperature $\left(T_{d}\right)$ of roughly $750 \mathrm{~K}$, ejecta temperatures $\left(T_{\mathrm{ej}}\right)$ of $7500 \mathrm{~K}$, and an $R_{\text {in }}=1.3 \times 10^{17} \mathrm{~cm}$ for this epoch. The blackbody temperature and radius of the IR component of SN 2010jl suggests pre-existing dust, since new dust formed in the CDS prior to day 90 would be expected to be much hotter, $\sim 1600 \mathrm{~K}$, as was the case in SN 2006jc and SN 2005ip and much closer to the expansion center, since the ejecta on day 90 would have traveled a maximum of $1.1 \times 10^{16} \mathrm{~cm}$ assuming a constant expansion of $14,000 \mathrm{~km} \mathrm{~s}^{-1}$ (Benetti et al. 2010). Any CDS would have to arise interior to this radius. Therefore, we believe that the IR emission most likely arises from pre-existing dust grains surrounding SN 2010jl heated by the initial flash.

As mentioned above, pre-explosion IRAC images do exist for UGC 5189A, but we are unable to detect the CSM shell surrounding the progenitor. This is not surprising if we assume either a red supergiant (RSG, $L=5 \times 10^{4} L_{\odot}, T=3500 \mathrm{~K}$ ) or an $\operatorname{LBV}\left(L=1 \times 10^{6} L_{\odot}, T=10,000 \mathrm{~K}\right)$ as a progenitor star, $48.9 \mathrm{Mpc}$ away with dust at a distance of $6 \times 10^{17} \mathrm{~cm}$; the total flux contribution from both the star and the dust in the pre-explosion image in 3.6 and $4.6 \mu \mathrm{m}$ is less than $0.5 \mu \mathrm{Jy}$.

In order to estimate the pre-existing dust mass in SN 2010j1, we used our radiative-transfer code MOCASSIN, a threedimensional Monte Carlo radiative-transfer code that is capable of modeling non-spherical geometries, clumpy density distributions, and non-central energy sources (Ercolano et al. 2003, 2005, 2008). As an initial fit, we used a "smooth" model, in which the dust is uniformly distributed throughout a spherical shell according to an $r^{-2}$ density profile, and constrained within a shell with size $R_{\text {in }}$ and $R_{\text {out }}$. We used a standard Mathis-Rumpl-Nordsieck grain size distribution of $a^{-3.5}$ between 0.005 and $0.05 \mu \mathrm{m}$ (Mathis et al. 1977), as was used in previous modeling done by Meikle et al. (2007), Kotak et al. (2009), and Andrews et al. (2011). Due to the early epoch of the observations, we assume the $\mathrm{SN}$ is a point source at the center of a spherical shell.

We used version 2.02.67 of MOCASSIN, which includes the capacity to account for light travel time effects (see Wesson et al. 2010 for details), to calculate the emission from only that portion of the dust illuminated after 90 days (Figure 4). When light travel times are taken into account, after 90 days only about $5 \%$ of a spherical shell with $R_{\text {in }}=6 \times 10^{17} \mathrm{~cm}$ and $R_{\text {out }}=$ $1.4 \times 10^{18} \mathrm{~cm}$ would be illuminated as seen from Earth (see Figure 4). Applying this constraint, spherical dust geometries could provide a good match to the observed day 90 SED, with a total dust mass of $\sim 0.6 M_{\odot}$, but gave a line-of-sight optical depth of $\sim 6$. This was problematic since previous papers from Patat et al. (2011) and Smith et al. (2011b) estimated a Galactic foreground reddening of $E(B-V)=0.027$ for SN 2010j1 and no reddening from the host galaxy. SN 2010jl is a good match to SN 2006tf, which also had little host galaxy reddening (Smith et al. 2011b). Patat et al. (2011), using both the continuum of the optical spectra and equivalent widths of the $\mathrm{NaI} \mathrm{D}$ lines, 


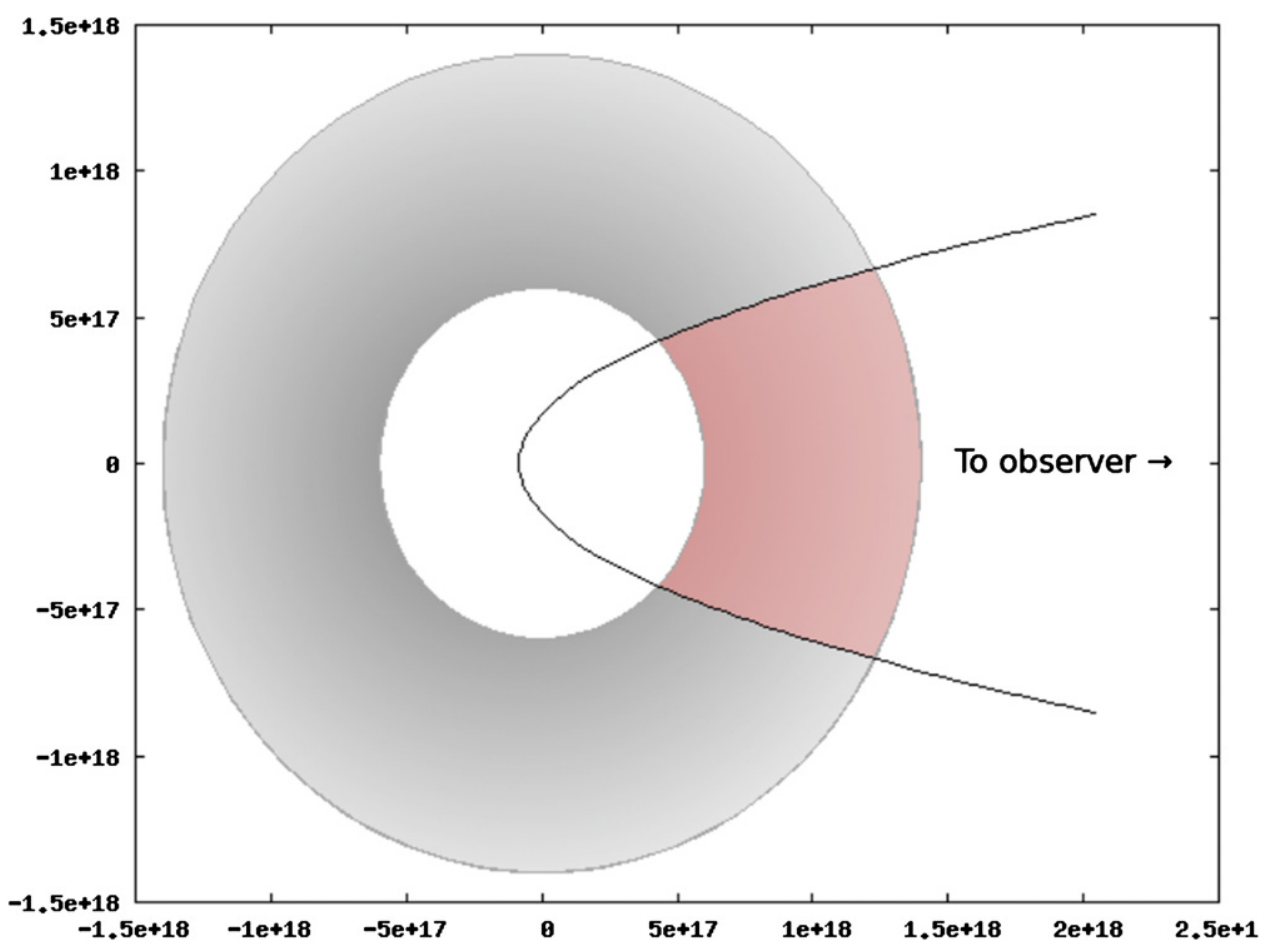

Figure 4. Schematic of the fraction of the CSM ejecta which will have been lit when light travel times are taken into consideration. For the example above, at 90 days, only about $5 \%$ would have been illuminated.

(A color version of this figure is available in the online journal.)

also found no host galaxy reddening. Therefore, a geometry other than a spherical shell, such as an inclined torus or bi-polar lobes, needs to be considered in order to allow low optical depth along the line of sight, but with sufficient dust mass to account for a CSM envelope with the high IR excess of SN 2010j1.

For the torus models we used a uniform dust density with a distance of 1.2 light years $\left(6 \times 10^{17} \mathrm{~cm}\right)$ and a tube radius of 0.5 light years $\left(4.7 \times 10^{17} \mathrm{~cm}\right.$; Ercolano et al. 2007). A torus with zero inclination (in the plane of the sky) would not have been illuminated by the $\mathrm{SN}$ by day 90 . An inclination of at least $45^{\circ}$ is required for IR emission to be present on day 90 . Figure 3 shows the fits for the $60^{\circ}$ inclination. We used both $100 \%$ amorphous carbon (AC) compositions and 100\% silicate ( $\mathrm{Si}$ ) compositions, since the lack of data beyond $4.5 \mu \mathrm{m}$ makes it impossible to differentiate between silicate and AC grains. We find an inner radius of $6 \times 10^{17} \mathrm{~cm}$ also provided the best fit to the data, which is actually larger (particularly for a carbon-rich CSM) than the predicted evaporation radius discussed above. This suggests that dust was absent interior to $r_{\text {evap }}$ before the explosion, and that the pre-existing dust was actually formed by a progenitor eruption as early as 300 years prior (if we assume an LBV eruption of $\sim 600 \mathrm{~km} \mathrm{~s}^{-1}$, as was seen in the bipolar lobes of $\eta$ Car; Smith et al. 2003) or as late as 20,000 years if we assume an RSG progenitor with a constant wind of $10 \mathrm{~km} \mathrm{~s}^{-1}$.

The $45^{\circ}$ inclined torus, although having a zero optical depth underestimates the IR flux. The $80^{\circ}$ torus does a fairly good job fitting the data, but has an optical depth of 1.53 and 2.4 for the $\mathrm{AC}$ and $\mathrm{Si}$ models, respectively, much too high for the reddening suggested by the early-time optical spectra. The $60^{\circ}$ torus includes both a zero optical depth and an acceptable fit to the data, making it the most likely inclination of the surrounding CSM torus. With only the two mid-IR points the exact inclination is not very well constrained, but an inclination of $60^{\circ}-80^{\circ}$ to the plane of the sky surrounding SN 2010jl is consistent with the data. This model (see input parameters listed
Table 4

Monte Carlo Radiative-transfer Torus Models for 100\% Amorphous Carbon

\begin{tabular}{lcccccc}
\hline \hline Inclination & $T_{\mathrm{ej}}(\mathrm{K})$ & $R_{\text {in }}(\mathrm{cm})$ & $R_{\text {out }}(\mathrm{cm})$ & $L_{\text {tot }}\left(L_{\odot}\right)$ & $\tau_{v}$ & $M_{d}\left(M_{\odot}\right)$ \\
\hline $45^{\circ}$ & 7500 & $6 \mathrm{e} 17$ & $1.4 \mathrm{e} 18$ & $5.5 \mathrm{e} 9$ & 0.0 & 0.12 \\
$60^{\circ}$ & 7500 & $6 \mathrm{e} 17$ & $1.4 \mathrm{e} 18$ & $5.5 \mathrm{e} 9$ & 0.0 & 0.05 \\
$80^{\circ}$ & 7500 & $6 \mathrm{e} 17$ & $1.4 \mathrm{e} 18$ & $5.5 \mathrm{e} 9$ & 1.5 & 0.03 \\
\hline
\end{tabular}

Table 5

Monte Carlo Radiative-transfer Torus Models for $100 \%$ Silicates

\begin{tabular}{lcccccc}
\hline \hline Inclination & $T_{\text {ej }}(\mathrm{K})$ & $R_{\text {in }}(\mathrm{cm})$ & $R_{\text {out }}(\mathrm{cm})$ & $L_{\text {tot }}\left(L_{\odot}\right)$ & $\tau_{v}$ & $M_{d}\left(M_{\odot}\right)$ \\
\hline $45^{\circ}$ & 7500 & $6 \mathrm{e} 17$ & $1.4 \mathrm{e} 18$ & $2.6 \mathrm{e} 10$ & 0.0 & 0.7 \\
$60^{\circ}$ & 7500 & $6 \mathrm{e} 17$ & $1.4 \mathrm{e} 18$ & $2.6 \mathrm{e} 10$ & 0.0 & 0.35 \\
$80^{\circ}$ & 7500 & $6 \mathrm{e} 17$ & $1.4 \mathrm{e} 18$ & $2.6 \mathrm{e} 10$ & 2.4 & 0.27 \\
\hline
\end{tabular}

in Tables 4 and 5) implies that between 0.03 and $0.35 M_{\odot}$ of pre-existing dust surrounds SN 2010jl. This would indicate total masses of CSM between 3 and $35 M_{\odot}$, assuming a gas-to-dust ratio of 100 and the possible inclinations and dust compositions. Although this is a large amount, measurements of dust around Eta Carinae, an extensively studied LBV in our Galaxy, yield dust values between 0.4 and $0.7 M_{\odot}$ (Gomez (Née Morgan) et al. 2006; Gomez et al. 2010) making it plausible that previous massloss events could have ejected this much dust around SN 2010j1. Like the geometry of $\eta$ Carinae, we cannot rule out the dust being contained in bipolar lobes and not in a torus. However, observations at later epochs will provide stronger constraints on the geometry of the dusty CSM which we have shown to exist around SN 2010jl.

\section{SUMMARY}

This paper presents the results of SED modeling of SN 2010j1 $\sim 90$ days post-explosion. Using optical, near-, and mid-IR 
observations of SN 2010jl from day 90 we have estimated a pre-existing dust mass between $M_{d}=0.03$ and $0.35 M_{\odot}$, with the higher dust masses corresponding to an oxygen-rich CSM. This dust is likely located in a torus inclined between $60^{\circ}$ and $80^{\circ}$ some $6 \times 10^{17} \mathrm{~cm}$ away from the SN. The inclined torus is required by the conclusion of Patat et al. (2011) and Smith et al. (2011b) that there is very little local reddening around SN 2010jl, while at the same time allowing the existence of substantial amounts of dust needed to create the observed IR excess. The lack of dust between the estimated evaporation radius and the inner dust radius suggests that this torus of pre-existing dust was created in a mass-loss episode likely 300-2000 years prior, assuming an LBV progenitor with expansion velocities between 100 and $600 \mathrm{~km} \mathrm{~s}^{-1}$. As many LBVs can have bipolar or toroidal nebulae and massive dust shells, it seems very likely that the progenitor was an LBV. This agrees with the conclusions of Smith et al. (2011b), who suggests the progenitor was a massive LBV star. If the inner edge of the CSM dust is $6 \times 10^{17} \mathrm{~cm}$ away, we estimate that the contribution to the elevated IR flux from pre-existing dust will last at least for 1.2 years, and that it in fact will increase as more and more of the torus is illuminated. Continued monitoring of this object is crucial in order to detect the formation of new dust, and to measure how much dust is created.

We thank the anonymous referee for the valuable suggestions that have improved this paper. This work has been supported by NSF grant AST-0707691 and NASA GSRP grant NNX08AV36H. This work was supported by Spitzer Space Telescope RSA 1415602 and RSA 1346842, issued by JPL/ Caltech. The standard data acquisition has been supported by NSF grants AST-0503871 and AST-0803158 to A. U. Landolt. We acknowledge with thanks the variable star observations from the AAVSO International Database contributed by observers worldwide and used in this research. D.L.W. acknowledges support from a Natural Sciences and Engineering Research Council of Canada (NSERC) Discovery Grant.

\section{REFERENCES}

Andrews, J. E., et al. 2010, ApJ, 715, 541

Andrews, J. E., et al. 2011, ApJ, 731, 47
Benetti, S., Bufano, F., Vinko, J., Marion, G. H., Pritchard, T., Wheeler, J. C., Chatzopoulos, E., \& Shetrone, M. 2010, CBET, 2536, 1

Cherchneff, I., \& Dwek, E. 2010, ApJ, 713, 1

Chevalier, R. A., \& Fransson, C. 1994, ApJ, 420, 268

Dwek, E. 1983, ApJ, 274, 175

Dwek, E. 1985, ApJ, 297, 719

Elmhamdi, A., et al. 2003, MNRAS, 338, 939

Ercolano, B., Barlow, M. J., \& Storey, P. J. 2005, MNRAS, 362, 1038

Ercolano, B., Barlow, M. J., \& Sugerman, B. E. K. 2007, MNRAS, 375, 753

Ercolano, B., Morisset, C., Barlow, M. J., Storey, P. J., \& Liu, X. 2003, MNRAS, 340, 1153

Ercolano, B., Young, P. R., Drake, J. J., \& Raymond, J. C. 2008, ApJS, 175, 534

Fox, O., et al. 2009, ApJ, 691, 650

Fox, O. D., et al. 2011, arXiv:1104.5012

Gal-Yam, A., \& Leonard, D. C. 2009, Nature, 458, 865

Gal-Yam, A., et al. 2007, ApJ, 656, 372

Gomez, H. L., Vlahakis, C., Stretch, C. M., Dunne, L., Eales, S. A., Beelen, A., Gomez, E. L., \& Edmunds, M. G. 2010, MNRAS, 401, L48

Gomez (Née Morgan), H. L., Dunne, L., Eales, S. A., \& Edmunds, M. G. 2006, MNRAS, 372, 1133

Kiewe, M., et al. 2010, arXiv:1010.2689

Kotak, R., et al. 2009, ApJ, 704, 306

Landolt, A. U. 2009, AJ, 137, 4186

Li, W., et al. 2011, MNRAS, 412, 1441

Mathis, J. S., Rumpl, W., \& Nordsieck, K. H. 1977, ApJ, 217, 425

Meikle, W. P. S., et al. 2007, ApJ, 665, 608

Meixner, M., et al. 2008, Proc. SPIE, 7014, 70142W

Newton, J., \& Puckett, T. 2010, CBET, 2532, 1

Patat, F., Taubenberger, S., Benetti, S., Pastorello, A., \& Harutyunyan, A. 2011, A\&A, 527, L6

Pozzo, M., Meikle, W. P. S., Fassia, A., Geballe, T., Lundqvist, P., Chugai, N. N., \& Sollerman, J. 2004, MNRAS, 352, 457

Schlegel, D. J., Finkbeiner, D. P., \& Davis, M. 1998, ApJ, 500, 525

Schlegel, E. M. 1990, MNRAS, 244, 269

Smartt, S. J., Eldridge, J. J., Crockett, R. M., \& Maund, J. R. 2009, MNRAS, 395,1409

Smith, N., Chornock, R., Silverman, J. M., Filippenko, A. V., \& Foley, R. J. 2010, ApJ, 709, 856

Smith, N., Davidson, K., Gull, T. R., Ishibashi, K., \& Hillier, D. J. 2003, ApJ, 586,432

Smith, N., Foley, R. J., \& Filippenko, A. V. 2008, ApJ, 680, 568

Smith, N., Li, W., Filippenko, A. V., \& Chornock, R. 2011a, MNRAS, 412, 1522

Smith, N., et al. 2009, ApJ, 695, 1334

Smith, N., et al. 2011b, ApJ, 732, 63

Stoll, R., Prieto, J. L., Stanek, K. Z., Pogge, R. W., Szczygiel, D. M., Pojmanski, G., Antognini, J., \& Yan, H. 2010, arXiv:1012.3461

Sugerman, B. E. K., et al. 2006, Science, 313, 196

Wesson, R., et al. 2010, MNRAS, 403, 474

Yamanaka, M., Okushima, T., Arai, A., Sasada, M., \& Sato, H. 2010, CBET, 2539,1 